\title{
SPLEENIC AND PULMONARY HYDATID CYST: A RARE SITE OF PRESENTATION
}

\author{
Umeshchandra $^{1}$, Avinash R. Odugoudar², Ankur Bhatia ${ }^{3}$
}

\section{HOW TO CITE THIS ARTICLE:}

Umeshchandra, Avinash R. Odugoudar, Ankur Bhatia. "Splenic and Pulmonary Hydatid CYST: A Rare Site of Presentation". Journal of Evolution of Medical and Dental Sciences 2014; Vol. 3, Issue 33, August 07;

Page: 8944-8948, DOI: $10.14260 /$ jemds/2014/3149

\begin{abstract}
Hydatid cyst of spleen with lung is a rare clinical entity. We report a case of a 5year old village girl, coming from agricultural family, living with pets (dogs) who presented with dragging sensation in the left hypochondrium and vague upper abdominal pain for the 15days. History and clinical examination gave the clinical suspicion of splenic and left pulmonary hydatid cyst. Ultrasonography and CT scan of the abdomen revealed two huge simple cyst in the spleen and the upper and middle lobe of left lung. Patient was treated by excision of cyst (laminar membrane) with pericystectomy and tube drainage of both the cysts. Preoperative in the left upper and middle lobe. A clinical diagnosis of cystic lesion of spleen with lung was made in the left upper and middle lobe. A clinical diagnosis of cystic lesion of spleen with lung was made. CONCLUSION: Although rare but a high degree of suspicion should be there when splenic cyst is encountered.
\end{abstract}

KEYWORDS: Spleen, pulmonary, pericystectomy, albendazole.

INTRODUCTION: Hydatid disease is endemic in this part of the world. Larvae of Echinococcus Granulosus cause hydatid disease in humans. It has a cosmopolitan distribution, being particularly prevalent in sheep and cattle raising areas of the world. ${ }^{1}$ Humans, being an incidental host, contract the disease by ingesting highly infective eggs of adult echinococcus harboring in the small intestine of the definitive hosts like dogs and canine animals. Human's ingestion of such contaminated food leads to hatching of ova in the gastrointestinal tract.

The enclosed embryos are liberated in the duodenum and transported to the liver via portal circulation. The liver acts as the first filter in trapping the embryos which then develop into hydatid cysts in $55-70 \%$ cases, followed by the lungs being 2 nd filter in $18-35 \%$ cases. Some escape from these to involve the brain, kidneys, bones and other soft tissues. ${ }^{1}$ Hydatid cysts involving the spleen are rare $(2.5 \%)$. Splenic hydatid, being a rare entity, can occur primarily or in association with Hepatic, Pulmonary or multi-organ hydatidosis. ${ }^{2}$

Hydatid cyst presents symptom according to involved organ. In this case report, pulmonary and splenic cyst hydatid case with nonspecific symptoms such as cough, chest pain, abdominal pain reflecting to left shoulder and fever and have radiologically suspected chest radiography, was presented with ultrasonography and computed tomography imaging, thereby reviewing literatures. ${ }^{1-3}$

\section{CASE REPORT:}

HISTORY: A 5 years old girl presented with 15days history of pain and heaviness in the left hypochondrium. The pain was localized, gradual in onset, moderate in intensity aching in character, associated with a feeling of heaviness and mass in upper abdomen. The patient is from a poor socioeconomic class and history of exposure to dogs is present. 


\section{CASE REPORT}

Physical Examination: Blood pressure of 100/ $60 \mathrm{mmHg}$, pulse 80 beats/min. and a temp. of $98.6 \mathrm{~F}$. On abdominal examination, there was a non-tender, irregular cystic mass moving with respiration and occupying whole of the left hypochondrium and lumbar region reaching upto umbilicus and extending $8 \mathrm{~cm}$ from Lt. costal margin. Respiratory system examination revealed retraction on the left hemithorax and absent air entry in the left upper and middle lobe. A clinical diagnosis of cystic lesion of spleen with left lung was made.

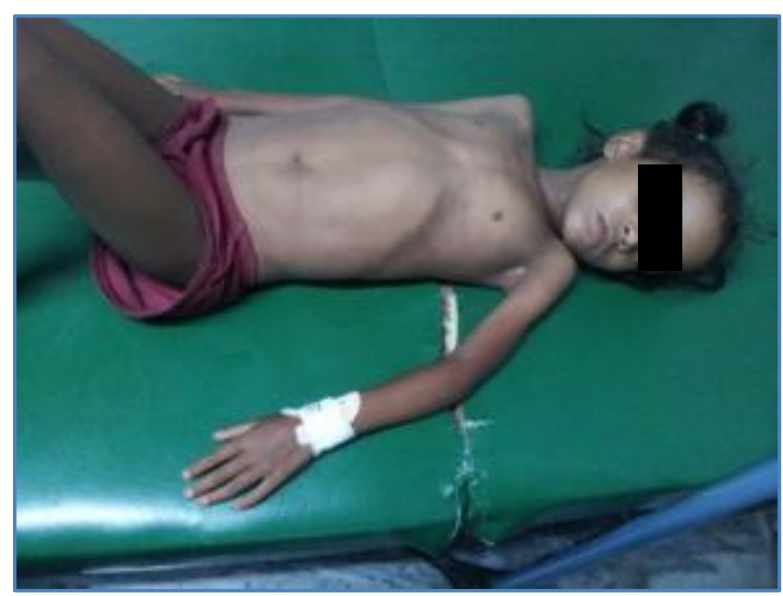

Fig. 1: Shows fullness in left hypochondrium

INVESTIGATIONS: TLC: was raised to16300; ESR: 60; Eosinophil count was high (14\%). Ultrasonography examination showed a simple cyst measuring $2 \times 13 \times 16.5 \mathrm{~cm}$ occupying whole of the spleen with another cyst occupying upper and middle lobe of left lung measuring was noted.
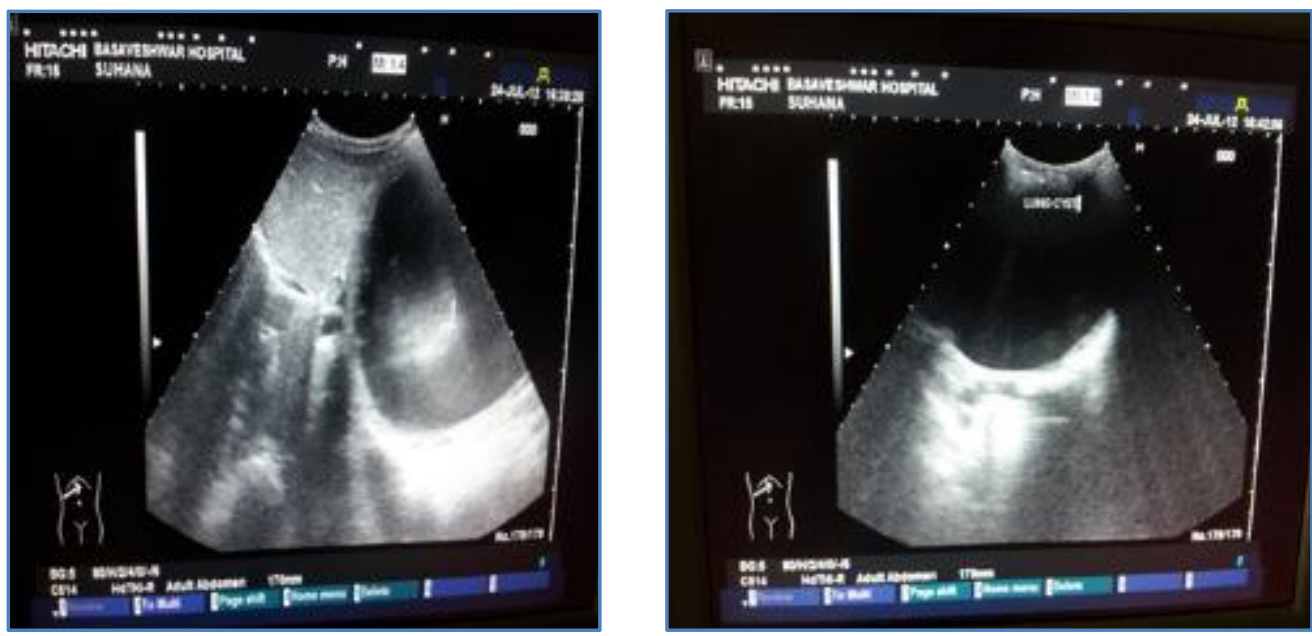

Fig. 2 \& 3: Ultrasonographic demonstration of the cystic cavity in the spleen and the middle lobe of left lung 


\section{CASE REPORT}

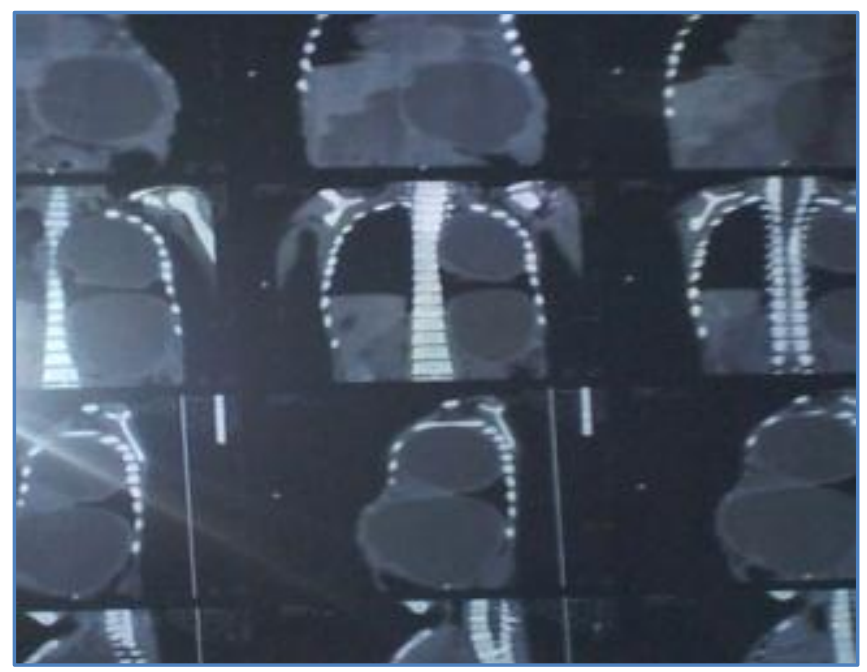

Fig. 4: CECT scan of chest and abdomen cystic cavity in spleen and middle lobe of left lung

TREATMENT: Patient was planned for surgical treatment. Preoperatively patient was vaccinated with pneumococcal and H. influenza b and treated with Tab Albendazole 200mg/day for 14days before surgery.

Exploratory laparotomy done through left sub costal incision revealed a huge cyst occupying the spleen. Excision of the laminar membrane with pericystectomy and tube drainage was performed. Similarly the pulmonary cyst was excised and drained with thoracotomy incision at $5^{\text {th }}$ intercostal space on the left side. Spleen and both the lobes of lungs were preserved. Postoperatively on day 2 patients was allowed orally and Tab.

Albendazole $200 \mathrm{mg} /$ day was continued for 12weeks. Repeat scan performed on day 6 showed the cyst cavities were collapsed and hence both the drains were removed on day8. The patient was discharged on 10th post-operative day in a satisfactory condition. Follow up was done after 6 month; patient was healthy and had no fresh complaints.
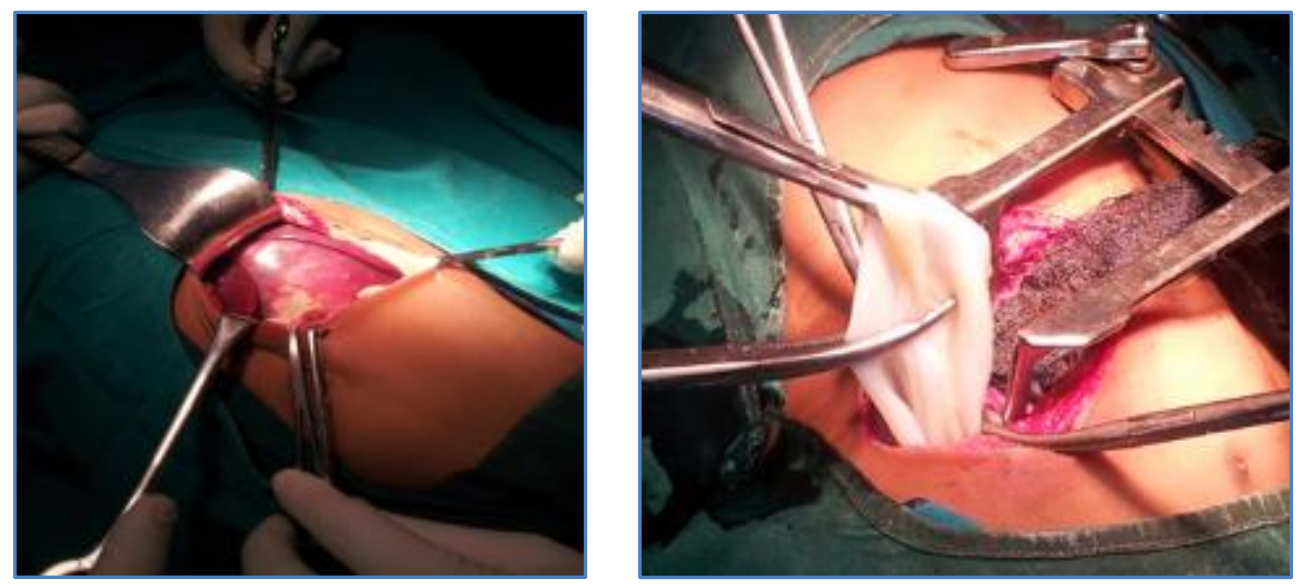

Fig. 5 \& 6: Intraoperative; 5 shows the hydatid cyst of spleen; 6 showing the laminar layer of hydatid cyst 
DISCUSSION: Splenic cysts are usually parasitic in origin and are mostly echinococcal, while nonparasitic cysts being categorized as dermoid, epidermoid and pseudocysts. ${ }^{4}$ Echinococcus granulosus causes cystic echinococcosis, Echinococcus multilocularis produces multilocular hydatid (alveolar echinococcosis) and other species like Echinococcus vogeli and oligarthus result in polycystic form. Infection is usually acquired in childhood but they mostly remain asymptomatic.

The cyst grows at a rate of $0.3-1 \mathrm{~cm}$ per year and it may take 5-20 years to grow into a sufficient size $(3-35 \mathrm{cms}$ ) to cause symptoms of a constant abdominal pain and a visible/palpable swelling in the abdomen as presenting complaints. ${ }^{5}$ Patients can even present with complications like rupture of cysts into the peritoneal cavity, gall bladder, biliary tree, pleural cavity or hepatic veins.

Trivial abdominal trauma can cause the rupture of huge body cysts and the patient can present with profound signs and symptoms which are not in keeping with injury. Moderate eosinophilia of $6 \%$ or more is usually present. Different serologic tests like hydatid immunophoresis, enzyme linked immunoassay (ELISA), Latex and indirect hemagglutination (IHA) test are helpful for the diagnosis, screening and post-op follow-up for recurrence. ${ }^{6}$

Preoperative diagnosis of this infection is mandatory in order to prevent any rupture of cyst so as to avoid anaphylactic shock or local recurrence. Since the condition closely resembles a soft tissue tumor on clinical examination, pre-operative radiological diagnosis is very important to avoid a biopsy. ${ }^{7}$ Plain films are usually incapable of showing cysts within a soft tissue. Preoperative diagnosis of hydatid cysts can be made on ultrasound and confirmed by CT scan (94-96\% and 100\% sensitivity respectively).Echocardiography and MRI are essential in cardiac and intracranial hydatidosis respectively. ${ }^{2}$

Treatment strategies include PAIR (puncture, aspiration, injection, re-aspiration) which was developed at the beginning of 1980s has proved to be successful in a variety of selected indications that have been reviewed by WHO recommendations. ${ }^{8}$

In the present case ultrasound scan done by a consultant radiologist showed a large unilocular cyst in the spleen and lung C.T Scan, added to the diagnostic accuracy by showing size, shape and extent of the lesion Surgery remains the standard treatment, however, pre and postoperative 1 month course of tab albendazole should be considered in order to sterilize the cyst and reduce the chances of anaphylaxis and tension in the cyst wall.

This reduces the risk of spillage during surgery and reduces the recurrence rate postoperatively. Medical treatment with albendazole is indicated in inoperable cases, in patients who refuse surgery and as an adjuvant therapy pre-operatively and post operatively to avoid recurrence. ${ }^{9}$

CONCLUSION: Hydatidosis should be considered in the existence of appropriate clinical and radiological findings as a probable diagnosis in all children in our region. Surgery should be the first choice for treatment but, medical therapy was considered as effective for treatment complicated and/or nonsurgical pediatric cases.

\section{REFERENCES:}

1. A Cuschieri, RJC Steele, AR Moosa. Infected patients: Essential surgical practice. 4th ed. Oxford. Butterworth-Heineann; 2000: p.157-60.

2. Murtaza B, Gondal ZI, Mehmood A, Shah SS, Abbasi MH, Tamimy MS, et al. Massive splenic hydatid cyst. JCPSP 2005 Sep; vol 15 (9): 568-70. 
3. Manouras AJ, Nikolauo CC, Katergiannakis VA. Spleen Sparing surgical treatment for echinococcosis of the spleen. Br J Surg 1997; 84: 1162.

4. Kir A, Baran E. Simultaneous operation for hydatid cyst of right lung and liver. Thorac Cardiovasc Surgeon 1995; 43: 62-4.

5. Wani NA, Tak S, Shah ND, Bashir A, Arif SM. Bullet injury causing rupture of spleen with hydatid Cyst. JK Pract 1998; 5: 55-56. Splenic hydatid, a rare presentation of hydatid disease annals vol 16. No. 2 apr. - jun. 2010131

6. Goel MC, Agarwal MR. Misra A. Percutaneous drainage of renal hydatid cyst: early results and follow up. Br J Urol 1995; 75: 724-8.

7. Kune GA, Morris D. Hydatid disease; Schwasyz and Ellis, eds. Maingot's Abdominal operations, 9th edn. Appleton \& Lange, 1989: 1225-40.

8. Maqbool, Anwar SF. Hepatic hydatid cyst presenting as anaphylaxis. JCPSP 2007 April; vol 17 (4): 224-25.

9. Bilal A, Saleem M, Nadeem A, Jan S, Nabi S. Surgical treatment of pulmonary hydatid disease. J postgrad Med Inst 2003; 17: 94-8.

\section{AUTHORS:}

1. Umeshchandra

2. Avinash R. Odugoudar

3. Ankur Bhatia

\section{PARTICULARS OF CONTRIBUTORS:}

1. Professor, Department of Surgery, Mahadevappa Rampure Medical College, Gulbarga.

2. Post Graduate, Department of Surgery, Mahadevappa Rampure Medical College, Gulbarga.

3. Post Graduate, Department of Surgery, Mahadevappa Rampure Medical College, Gulbarga.

\section{NAME ADDRESS EMAIL ID OF THE CORRESPONDING AUTHOR:}

Dr. Avinash R. Odugoudar, Uday Nagar, Mulgund Naka, Hubli Road, Gadag-582101, Karnataka.

Email: draviii86@gmail.com

Date of Submission: 16/07/2014. Date of Peer Review: 17/07/2014. Date of Acceptance: 31/07/2014. Date of Publishing: 06/08/2014. 